\title{
Review of: "Heterozygous transcriptional and nonsense decay signatures in blood outgrowth endothelial cells from patients with hereditary haemorrhagic telangiectasia"
}

\author{
Luisa Maria Botella ${ }^{1}$ \\ 1 Centro de Investigaciones Biológicas
}

Potential competing interests: The author(s) declared that no potential competing interests exist.

The paper represents an experimental demonstration of nonsense mediated RNA decay mutations in endothelial cells of patients suffering from HHT1, HHT2 and JPHT. This paper is the only experimentally based evidence published on this subject in HHT.

It represents the collection of enormous experimental work, based on the RNAseq technique, to show the differential gene expression of HHT cells with stop codon mutations, compared to wild type endothelial cells.

It is very important to stress the difficult task of obtaining primary cultures derived from HHT patients. On top of that, the cells were grown from patients containing nonsense heterozygous mutations in Endoglin (HHT1), ALK1/ACVRL1 (HHT2) and SMAD4/MADH4 (JPHT), conditions that require time and endurance in the experimental work.

Although very difficult to show, the authors tackle and show to some extent, how transcripts harbouring premature termination codons undergo nonsense mediated decay in blood endothelial cells from HHT patients. The percentage of estimated loss for the nonsense heterozygous mutant is of $89 \%$. This is a result not estimated/published before, and derived from very precise experiments and bioinformatics analysis.

Enrichment of genes for endoplasmic reticulum stress and the unfolded protein response in the GO term is an unexpected finding, but relevant in agreement with the HHT BOECs with nonsense alleles in highly expressed endothelial genes (Endoglin, ALK1, SMAD4). However it is also shown that Nonsense Mediated Decay (NMD) of mutant transcripts is not $100 \%$ efficient. Mutant transcripts could escape NMD. Pulse chase experiments support in part this result. The difficulty in the western blots (WB) resulting from pulse chase experiments is due to time constraints. However, it is possible to observe that partially decayed nonsense alleles were generating mutant protein and therefore, aberrant protein maturation kinetics. Those aberrant proteins are retained in the endoplasmic reticulum (ER) leading to cellular responses in relation to ER stress. As an alternative to the WB, it could be possible to see the aberrant proteins by confocal fluorescent microscopy in the ER. This is only a suggestion to perhaps visualize these immature aberrant proteins. 
In conclusion the paper is the result of a huge experimental work which measures, and shows for the first time the NMD process in HHT with nonsense mutations. The message of the paper helps to understand the role of nonsense mutations as null alleles, and perhaps the use of some drugs like ataluren to partially skip this nonsense signals.

Congratulations for the huge work! 